\title{
Occurrence of a 5,7-Diene Sterol in Leishmania donovani
}

\author{
By S. S. KUWAHARA \\ Department of Chemistry, California State College at Long Beach \\ AND \\ L. J. PINTO AND B. H. KAZAN \\ Department of Microbiology, California State College at Long Beach, \\ Long Beach, California 90801, U.S.A.
}

(Accepted for publication Io May 1971)

Ergosterol, a 5,7-diene sterol, has been isolated from a number of the Trypanosomidae. It is the major sterol in Crithidia fasiculata (Kusel \& Weber, 1965) and is found in related organisms including Leishmania tarentolae (Meyer \& Holz, 1966) and Leptomonas culcididarum (Halevy \& Sarel, I965). Halevy (1962) also showed the presence of 'fast-acting' sterols in Trypanosoma cruzi and Leishmania tropica.

Williamson \& Ginger (1965) found traces of 5,7-diene sterols in several Trypanosoma species but did not detect any in Leishmania donovani (Williamson, 1963; Williamson \& Ginger, I965) and concluded that cholesterol is the major sterol in the organism. However, Ghosh (1963) working with membranes from $L$. donovani, found a sterol which was 'fastreacting' with the Lieberman-Buchard reagent indicating the presence of a $\Delta^{7}$ or $\Delta^{8}$ structure (Cook \& Rattray, I958), but did not obtain an ultraviolet spectrum showing the presence of a 5,7-diene and concluded that 5,7-dienes and cholesterol were absent.

Our studies on the lipids of Leishmania donovani have produced results contrary to those reported by Williamson (1963) and we have obtained an ultraviolet spectrum confirming the presence of 5,7 -dienes in the sterol fraction from $L$. donovani.

\section{METHODS}

Leishmania donovani (National Institutes of Health, U.S.A., strain, obtained from Dr E. J. Tobie) was cultured in a diphasic medium consisting of an agar supplemented brain-heart infusion agar (Difco) containing $2 \%(\mathrm{w} / \mathrm{v})$ glucose and $10 \%(\mathrm{v} / \mathrm{v})$ defibrinated rabbit blood as the solid base. Locke's solution was used as an overlay. The cultures were grown at room temperature for 8 to Io days before harvesting by centrifugation at $3500 \mathrm{~g}$ for $10 \mathrm{~min}$. at room temperature.

The harvested organisms were suspended in 0.1 culture volumes of $100 \mathrm{mM}-\mathrm{KCl}$ at $5^{\circ}$ and recentrifuged. The washed organisms were resuspended in 0.0 I culture volume of cold I00 $\mathrm{mM}$ KCL and extracted by the method of Bligh \& Dyer (1959).

The dry, total lipid extract from $10^{10}$ organisms was added to a $15 \mathrm{~g}$. column of silicic acid (CC-4; Mallinkrodt Chemical) and the neutral lipids were eluted with chloroform. Chloroform was removed with a rotary evaporator and the neutral lipids were dissolved in hexane. The sample was then chromatographed on a $10 \mathrm{~g}$. column of Florisil (Floridin Co., Tallahassee, Florida) using the elution scheme given by Carroll (196I). The sterols were eluted with $15 \%(\mathrm{v} / \mathrm{v})$ diethyl ether in hexane.

Thin-layer chromatography was performed on silica gel G (Merck, Darmstat, Germany). 
A solvent system consisting of petroleum ether $\left(\mathrm{BP} ; 30^{\circ}\right.$ to $\left.60^{\circ}\right)+$ diethyl ether + acetic acid (70:30: I, by vol.) was employed for identifying and checking the purity of the sterol fraction and for detecting fatty acids in the lipid extract.

Six other solvent systems, known to separate various sterol groups, were used to determine the homogeneity of the sterol fraction on silica gel G (Lisboa \& Diezfalusy, I962; Elattar, I965). In all cases only a single spot was observed, indicating a homogeneous preparation at least with respect to the sterol class.

\section{RESULTS}

Thin-layer chromatography of the lipid extract showed the absence of free fatty acids indicating that sterol esters, glycerides, and phospholipids had not been degraded during preparation and extraction of the organisms.

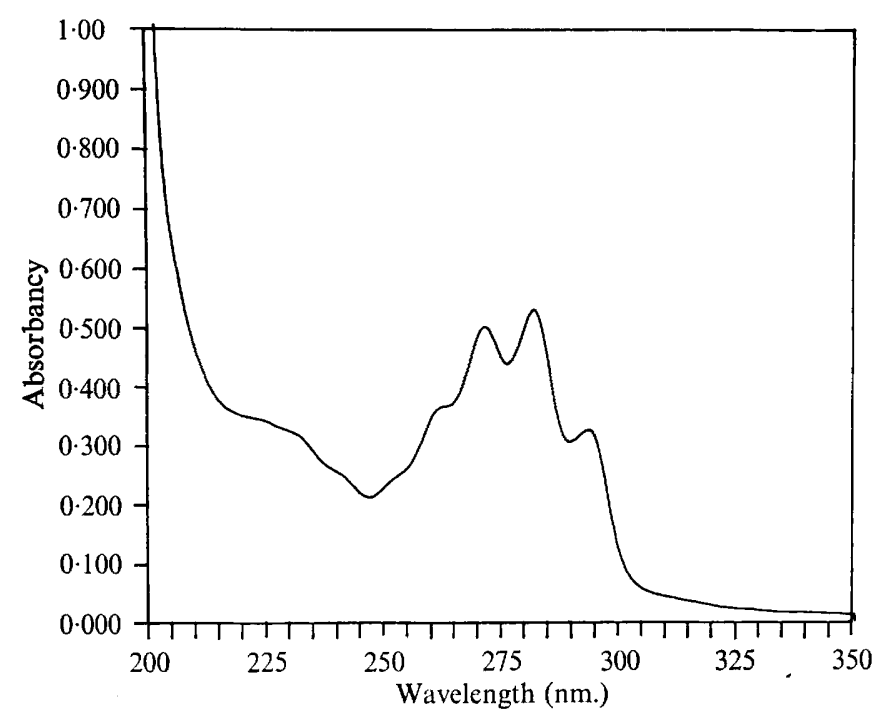

Fig. I. Ultraviolet spectrum of a diluted ethanol solution of Leishmania donovani sterol. $\lambda_{\max }: 272 \mathrm{~nm}$., $283 \mathrm{~nm}$. and $293 \mathrm{~nm}$.

Qualitative analysis using a Leiberman-Burchard reagent (Ness, Pastewka \& Peacock, 1964) gave full colour development after I min. of reaction with the Leishmania donovani sterol which agreed with the time required to develop the colour of an ergosterol control sample. The cholesterol control sample required about $20 \mathrm{~min}$. for full colour development with this system.

The sterol fraction obtained from 41 . of culture containing about $10^{10}$ organisms was dissolved in $\mathrm{I} 0 \mathrm{ml}$. of absolute ethanol and a $\mathrm{I}: 300$ dilution of this sample was scanned in a Beckman DK-2A spectrophotometer using an ethanol blank. The ultraviolet spectrum obtained is shown in Fig. I. The spectrum shows absorption maxima at 272, 283 and $293 \mathrm{~nm}$., with a shoulder at $263 \mathrm{~nm}$; these are characteristic of ergosterol and other 5,7-diene sterols (Fieser \& Fieser, 1959). The absorption in the region about $230 \mathrm{~nm}$. represents a yellowish contaminant that forms in the sterol preparations during storage. This material has been separated from the sterol fraction on a silicic acid column and has been found to absorb strongly with a band from 240 to $220 \mathrm{~nm}$. 
Our results, combined with the digitonin precipitate obtained by Ghosh (1963), indicate that the major sterol of the promastigote stage of Leishmania donovani is a 5,7-diene-3 $\beta$-ol. However, Williamson did not detect the 5,7-diene structure in $L$. donovani (Williamson, I963; Williamson \& Ginger, 1965) despite the use of both ultraviolet methods and Fieser's selenium dioxide reagent (Fieser, 1953).

It is difficult to evaluate the conflicting results reported in the literature with respect to the sterol composition of some of the Trypanosomidae for several reasons:

(I) Age of the culture. Leishmania, like many micro-organisms, would be expected to undergo metabolic changes while passing through various growth stages. Korn, von Brand \& Tobie (I969) have shown that the sterol composition of Trypanosoma cruzi changes as the culture ages. The present authors used cultures that had just entered the stationary phase of growth, and this may account for the observed difference between their results and those of other workers who may have utilized cultures from the log or death phases.

(2) Origin of the culture. The study presented in this paper was performed on a wellestablished culture strain of Leishmania donovani, while the work reported by Williamson (1963) was performed on a strain that had been freshly isolated from a hamster. Adaptation to culture conditions in vitro is known to influence biological factors such as virulence, and it is likely that the biochemical composition of the organisms would be also affected.

(3) Type of medium used for growth. The present authors and Ghosh (1963) cultivated the promastigote of Leishmania donovani in diphasic media. In these media the nutrients supplied by blood are initially contained in the solid phase and a preincubation period is required before inoculation in order that the liquid phase may support growth of $L$. donovani. This requirement for the diffusion of nutrients from the solid phase may result in a severe limitation of the amount of blood sterol (cholesterol in this instance) that is available for uptake by the organism. Under these conditions a well-established culture strain, such as that used in this study, would probably respond by synthesizing sterols de novo rather than depending upon assimilation from the surrounding medium to provide the sterols necessary for formation of cell membranes. The sterol composition of $L$. donovani that results from biosynthesis may thus be different from that which would result from direct incorporation of sterols from the medium. An example of this phenomenon was observed by Williams, Goodwin \& Ryley (I966), who reported that the epimastigote of Trypanosoma rhodesiense, when grown on diphasic media, produced ergosterol, while the blood stream trypomastigote form of this same organism, obtained from infected rats, contained cholesterol.

In our work we have found that the sterol fraction is extremely unstable when dry and rapidly develops a yellowish colour when exposed to air and light at room temperature. Dissolving the sterol fraction in ethanol retarded decomposition but did not eliminate it.

Recent studies by Korn, von Brand \& Tobie (1969) have shown that Trypanosoma cruzi and Crithidia fasiculata, when grown on serum or defined media, are capable of producing several different sterols with the 5,7-diene structure in addition to ergosterol, which was expected. These findings in closely related organisms suggest that the 5,7-diene in Leishmania donovani may not be ergosterol but a mixture of several related sterols.

We wish to thank Mrs Eleanor Tobie for the culture of Leishmania donovani. This work was supported in part by a grant from the Long Beach California State College Foundation to S.S.K. 


\section{REFERENCES}

BLIGH, E .G. \& DyER, W. J. (1959). Total lipid extraction and purification. Canadian Journal of Biochemistry and Physiology 37, 91 I-917.

CARROLL, K. K. (I96I). Separation of lipid classes by chromatography on florisil. Journal of Lipid Research 2, I35-I4I.

CooK, R. P. \& RatTRAY, B. M. (1958). Methods of isolation and estimation of sterols and appendix of practical methods. In Cholesterol, pp. 127, 490. Edited by R. P. Cook. New York: Academic Press.

Elattar, T. M. (1965). Early Metabolism in vivo of $\left[4^{-14} \mathrm{C}\right]$ androst-4-ene-3,17-dione in rat liver. Biochimica et biophysica acta III, 299-305.

FieSER, L. F. (1953). Cholesterol and companions. III. Cholestanol, lathosterol, and ketone I04. Journal of the American Chemical Society 75, 4395-4403.

FIESER, L. F. \& FIESER, M. (1959). Steroids, Ist edn, p. 16. New York: Reinhold.

GHOSH, B. K. (1963). Isolation and characterization of the membrane of Leishmania donovani. Annals of Biochemistry and Experimental Medicine 23, 159-168.

HALEVY, S. (1962). Comparative studies of lipid metabolism of Trypanosomidae. I. Trypanosoma cruzi, Leishmania tropica and related species. Bulletin of the Research Council of Israel 1o E 2, 65-68.

HALEVY, S. \& SAREL, S. (1965). Isolation of ergosterol from the trypanosomatid Leptomonas culcidarum. Journal of Protozoology 12, 293-296.

Korn, E. D., BRAND, T. von \& TOBIE, E. J. (1969). The sterols of Trypanosoma cruzi and Crithidia fasiculata. Comparative Biochemistry and Physiology 30, 601-6ro.

KuSEL, J. P. \& WeBER, M. M. (1965). Coenzyme Q9 (ubiquinone (45)) and ergosterol in Crithidia fasiculata Biochimica et biophysica acta 98, 632-639.

LisBoA, B. P. \& Diezfalusy, E. (1962). Separation and characterization of steroid estrogens by means of thin-layer chromatography. Acta endocrinologica 40, 60-81.

MEYER, H. \& HoLz, G. G. JUN. (1966). Biosynthesis of lipids by kinetoplastid flagellates. Journal of Biological Chemistry 24I, 5000-5007.

Ness, A. T , Pastewka, J. V. \& Peacock, A. C. (1964). Evaluation of a recently reported stable LiebermanBurchard reagent and its use for the direct determination of serum total cholesterol. Clinica chimica acta I0, 299-337.

Williams, B. L., Goodwin, T. W. \& Ryley, J. F. (1966). The sterol content of some protozoa. Journal of Protozoology 13, 227-229.

Williamson, J. (1963). The chemical composition of trypanosomes. Proceedings of the XVI International Congress of Zoology, Washington, D.C., pp. 189-195.

WiLliamson, J. \& Ginger, C. D. (1965). Lipid constitution of some protozoa, spirochaetes and bacteria. Transactions of the Royal Society of Tropical Medicine and Hygiene 59, 366-367. 